\title{
Controlling Ant-Based Construction
}

\author{
Lenka Pitonakova $^{1,2}$ and Seth Bullock ${ }^{1}$ \\ ${ }^{1}$ Institute for Complex Systems Simulation \\ University of Southampton, United Kingdom \\ 21.pitonakova@soton.ac.uk
}

\begin{abstract}
This paper investigates the dynamics of decentralised nest construction in the ant species Leptothorax tuberointerruptus, exploring the contribution of, and interaction between, a pheromone building template and a physical building template (the bodies of the ants themselves). We present a continuous-space model of ant behaviour capable of generating ant-like nest structures, the integrity and shapes of which are non-trivially determined by choice of parameters and the building template(s) employed. We go on to demonstrate that the same behavioural algorithm is capable of generating a somewhat wider range of architectural forms, and discuss its limitations and potential extensions.
\end{abstract}

\section{Introduction}

When building their nests, insect colonies are capable of creating extremely complex structures without employing explicit blueprints (Theraulaz et al., 1998). Such feats of collective construction are achieved via stigmergy, where deposition of building material and pheromones attract, guide and stimulate other nest mates. For example, some ant species progressively encircle their brood with a wall constructed from collected stones (Franks et al., 1992), while paper wasps build structured combs that are sometimes protected by an external envelope (Jeanne and Bouwma, 2002), and termites are capable of building highly complex nests with ventilation shafts, galleries, brood chambers, fungus gardens and royal chambers (Bonabeau et al., 1998; Ladley and Bullock, 2004, 2005).

Due to the inherent parallelism and stochasticity of selforganisation, insect nest construction is prone to problems of interference and needless redundancies are often created (Di Marzo Serugendo et al., 2011). Nevertheless, the fact that the individuals themselves only require limited sensors, memory and reasoning (Mason, 2002) makes attempting to reproduce their behaviour in robots attractive (Holland and Melhuish, 1999; Parker and Zhang, 2006; Bullock et al., 2012). In the future, we might be able to rely on swarms of extremely simple and cheap robots to autonomously build structures guided by environmental cues. For example, we could place signal beacons to suggest where corners of a building should be or where space should be created for windows and doors.

However, in order to be able to control insect-like construction, we must first understand it. This paper investigates nest building by the ant Leptothorax tuberointerruptus, which creates circular structures with one or more entrances around its brood. These nests are created inside flat horizontal cavities and can thus be studied in two dimensions (Franks et al., 1992; Franks and Deneubourg, 1997; Theraulaz et al., 2003). Ant builders can be divided into 'internal' and 'external' types. Internal ants stay in close proximity to the central brood cluster and tend to push stones away from it, while external ants repeatedly search for stones in the environment and push them directly towards the brood cluster until they collide with another ant or a wall.

The brood cluster and the internal ants that surround it serve as a physical template for construction, passively preventing stones from being pushed close to the brood cluster and actively moving stones away from it. Eventually, the built structure itself becomes more important for stigmergy and new stones are often bulldozed into or along existing walls. Building occurs in parallel in several places at once, with some stones travelling between building sites as different ants pick them up and drop them.

There is a certain ambiguity in the literature concerning the role of a pheromone template that emanates from the brood cluster. It is clear that ants use it to orient themselves within the nest (Franks et al., 1992), but it is not yet empirically established whether pheromone influences stone deposition directly (Franks and Deneubourg, 1997) leading existing models to differ in how they treat this aspect of ant behaviour (Franks et al., 1992; Theraulaz et al., 2003).

Furthermore, while global colony behaviour is well described (Franks et al., 1992) and modelled analytically (Franks and Deneubourg, 1997), existing agent-based simulations either use grid worlds where noisy movement and bulldozing with friction are not modelled (e.g. Franks et al., 1992) or implement simple continuous behaviour where ants are only of one type (e.g. Theraulaz et al., 2003). Moreover, Theraulaz et al. (2003) also model collisions abstractly, ide- 
alising ant stone-dropping behaviour as influenced directly by the local density of stones as well as local pheromone concentration.

Here, we attempt to achieve a better understanding of how the radius and integrity of the constructed nest result from the physical interactions between ants, stones and pheromone in a continuous-space model, where some of the shortcomings of the previous models are addressed. In particular, the following hypotheses are tested:

1. Nests will be larger when there are more internal ants in the colony and smaller when there are more external ants.

2. Using pheromone as a building template will lead to more regular structures, but will also interfere with (1), above.

3. Nest entrances will form spontaneously, influenced by the density of the internal ants and facilitated by the pheromone template.

We also explore extensibility of the building behaviour:

4. It is possible for the same behavioural algorithm to generate alternative architectures by combining multiple pheromone clouds.

\section{Methods}

All simulations were performed in a two-dimensional continuous-space arena of $660 \times 660$ units $\left(165 \mathrm{~mm}^{2}\right.$, i.e. 1 unit $=0.25 \mathrm{~mm}$ ). Objects were scaled proportionate to their real-world counterparts using dimensions given by Franks and Deneubourg (1997). At the beginning of each run, $3 \times 10^{3}$ rectangular stones of size $2 \times 2$ units $\left(0.5 \mathrm{~mm}^{2}\right)$ were placed randomly in the arena. The brood was represented by a tight cluster of 50 randomly oriented static ant agents placed around the middle of the arena in a random Gaussian fashion. A circular pheromone cloud 300 units in diameter was centred on the brood cluster such that the pheromone concentration had a constant value of unity at the centre, linearly decreasing to zero at the edge of the cloud. Time was modelled in discrete 0.02-second timesteps and each simulation run lasted 6000 seconds. All results presented in this paper are based on 20 runs per plotted data point.

Initially, a number, $N_{i}$, of 'internal' and, $N_{e}$, 'external' 10 units $\times 2$ units $(2.5 \mathrm{~mm} \times 0.5 \mathrm{~mm})$ ants were placed around the brood cluster. Ant location, orientation and movement were simulated as continuous (Bourg and Seemann, 2004, p.16-19), where the centre of an ant's body was moved by a real-valued distance and given a real-valued orientation each time step. Collision detection prevented ants from moving over or through stones or other ants including brood members.

Modelled ants implemented the following empirically observed behaviours (Franks et al., 1992) by executing the algorithm represented in Figures 1-3:

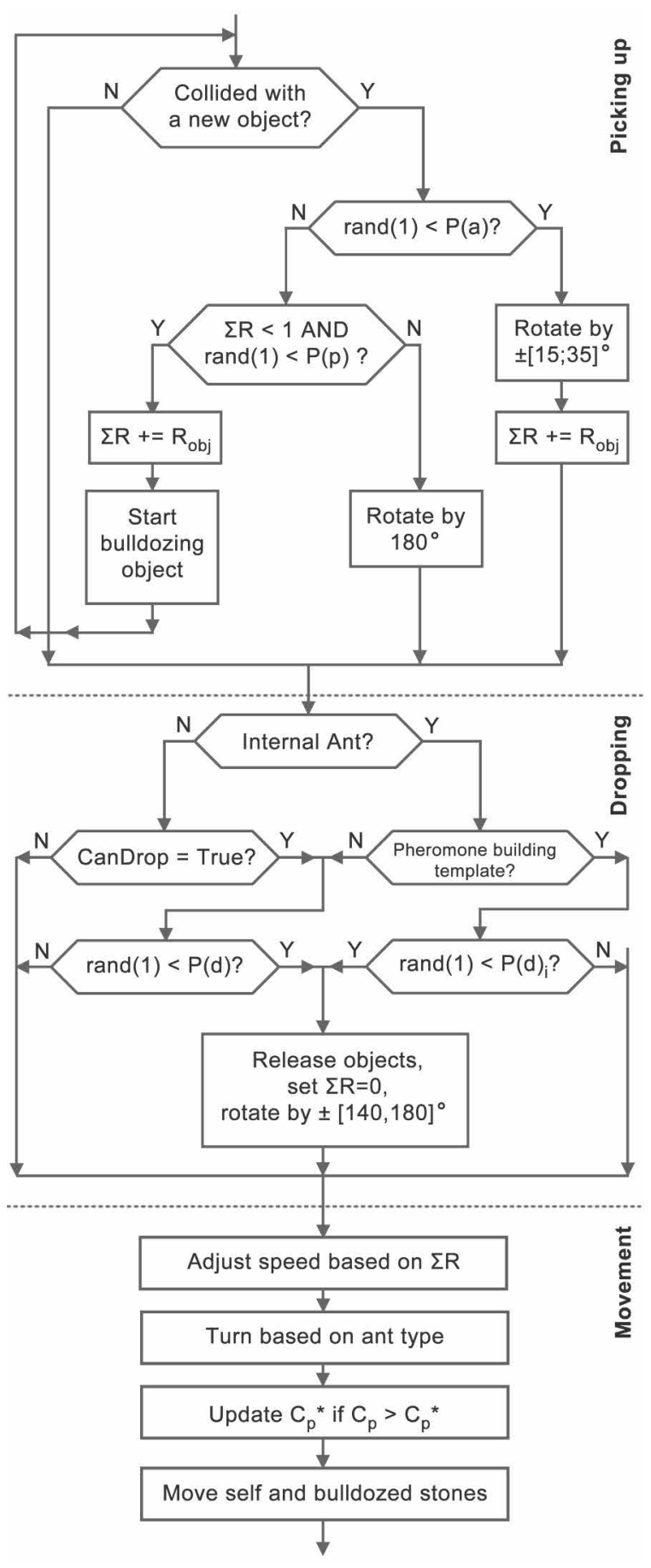

Figure 1: Ant behavioral cycle

1. Random movement, unless:

- bulldozing towards the brood cluster (external ants)

- bulldozing away from the brood cluster (internal ants)

- moving towards the brood cluster when pheromone levels were below a threshold (internal ants only) 


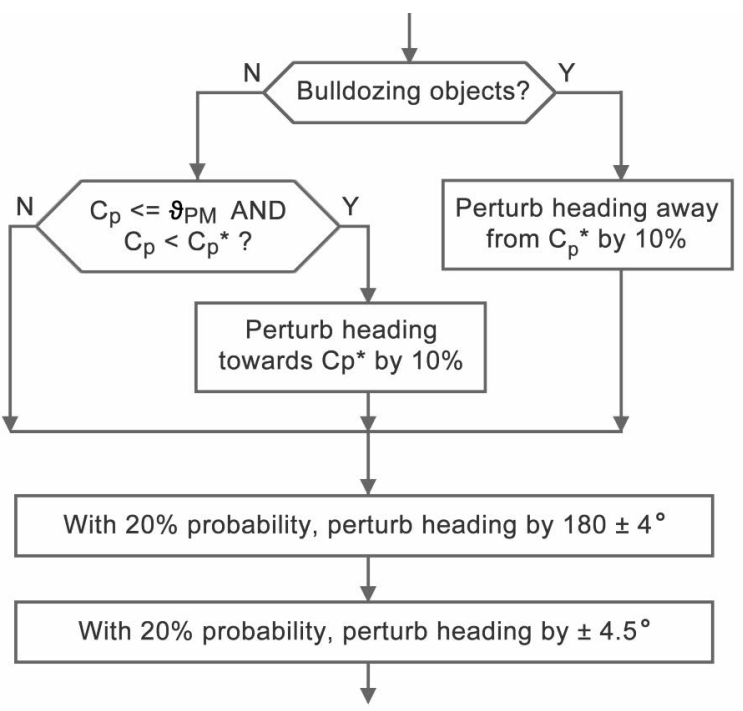

Figure 2: Internal ant's 'Turn based on ant type' routine

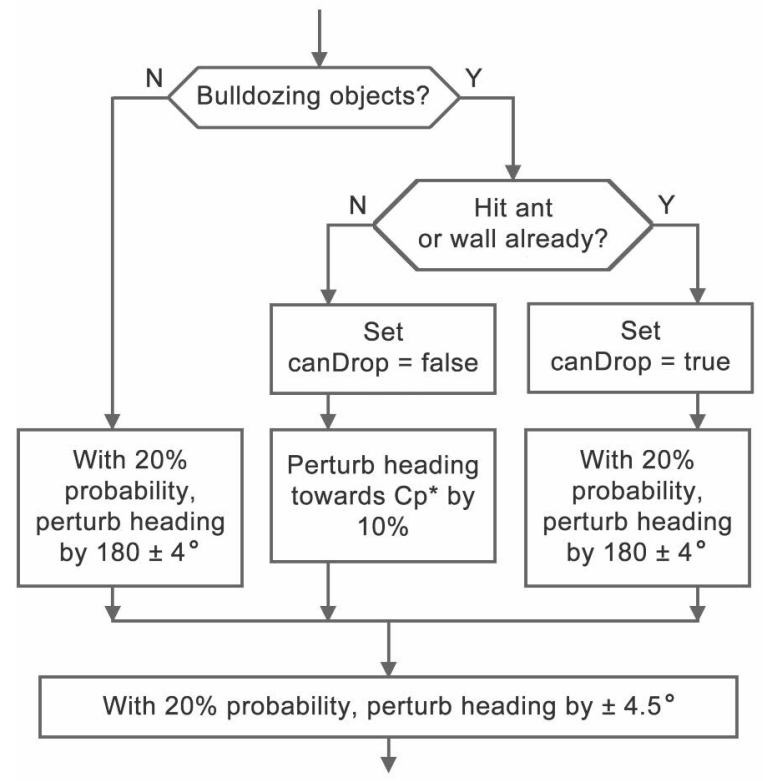

Figure 3: External ant's 'Turn based on ant type' routine

2. Stone bulldozing, i.e. pushing one or more stones forward

3. Stone dropping, the probability of which increased with felt resistance of whatever was being pushed or collided with

4. Occasional moving along walls while bulldozing. In this case, an ant rotated by a small value as it approached an existing wall and continued its movement along it.

In order to orient either towards or away from the brood cluster, both ant types relied on the ability to sense local pheromone concentration, $C_{p}$, and on remembering the location of the highest pheromone concentration that they had encountered so far, $C_{p} *$ : a proxy for the location of the brood cluster.

Internal ants (Figure 2) employed a pheromone movement threshold, $\vartheta_{P M}$, in order to remain within a characteristic distance of the central brood cluster. Where $\vartheta_{P M}=0$, internal ants were free to roam to the edge of the pheromone cloud. Where $\vartheta_{P M}=1$, internal ants attempted to remain as close to the brood cluster as possible. Internal ants tended to bulldoze encountered stones away from the brood cluster and then returned back. External ants (Figure 3) moved randomly unless they were bulldozing stones towards the brood cluster.

The probability of picking up stones when encountered was a constant $P(p)=0.5$. Each bulldozed stone or stone that an ant was currently colliding with added resistance $R_{s}=0.15$ to the ant's total felt resistance $\Sigma R \in[0,1]$. The resistance experienced during collisions with other ants was $R_{a}=1.0$.

When an ant pushed more stones, its speed decreased (Equation 1), while its probability to move along walls $P(a)$ and to drop stones $P(d)$ increased (Equations 2 and 3), causing the colony to gradually extend existing walls rather than to make them thicker. Note that external bulldozers only started checking whether they should drop stones after they encountered an immovable obstacle and turned away from it, while internal bulldozers could drop stones at any time.

$$
\begin{aligned}
& \text { speed }=2 \times(1-\Sigma R) \\
& P(a)=\Sigma R \\
& P(d)=f \times \mid \log (1-\alpha \times(\Sigma R+\epsilon) \mid ; \\
& f=0.625, \alpha=0.8, \epsilon=10^{-11}
\end{aligned}
$$

In runs where the pheromone building template was employed, local pheromone concentration, $C_{p}$, influenced each internal ant's drop probability, $P(d)_{i}$, such that it exponentially increased as the ant moved towards edges of the pheromone cloud (Equation 4).

$$
\begin{aligned}
& P(d)_{i}=\min \left(1, P(d)+\left|g \times \log \left(C_{p}\right)\right|\right) ; \\
& g=1 / 7
\end{aligned}
$$

The combined effect of $\Sigma R$ and $C_{p}$ on $P(d)$ and $P(d)_{i}$ is depicted in Figure 4.

\section{Results}

Structures built by the artificial ants were generally circular (Figure 5), with (sometimes incomplete) walls forming around the brood cluster at a characteristic distance that varied with model parameters. This result was robust with respect to the colony size and suitable values of the pheromone movement threshold, $\vartheta_{P M}$, and is comparable with the real and simulated ants in the existing literature (Franks et al., 1992; Theraulaz et al., 2003). 


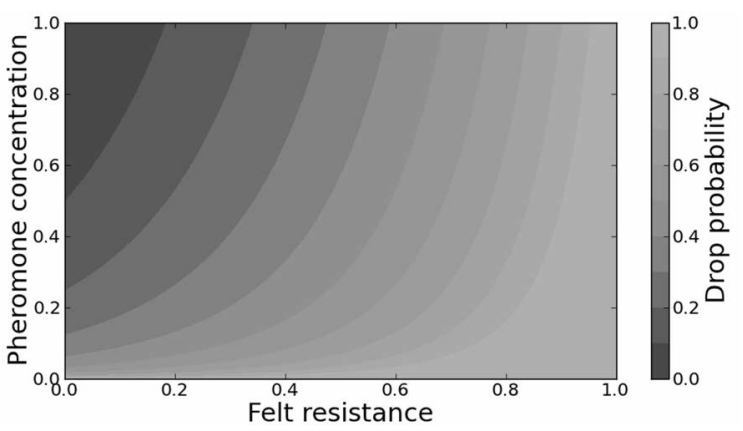

Figure 4: Contour plot of drop probability $P(d)_{i}$ as a function of felt resistance, $\Sigma R$, and local pheromone concentration, $C_{p}$, based on Equations 3 and 4 . In the absence of the pheromone building template, values of $P(d)$ are dependant on Equation 3 alone and are plotted at $y=1$, i.e. where the $\log$ of pheromone concentration is zero.

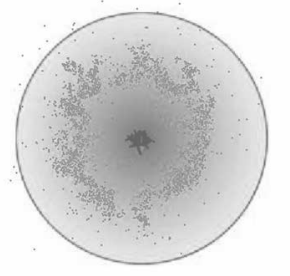

(a)

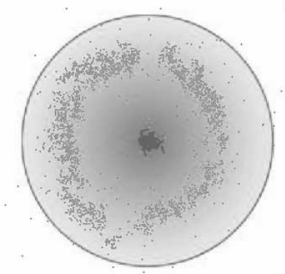

(b)

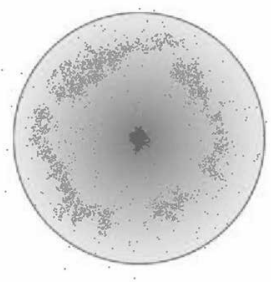

(c)

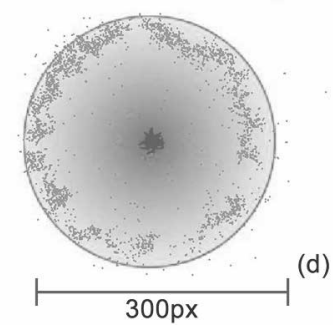

Figure 5: Example nests for various ant parameter combinations: (a) $N_{i}=10, \vartheta_{P M}=1.0$; (b) $N_{i}=30, \vartheta_{P M}=0.75$; (c) $N_{i}=50, \vartheta_{P M}=1.0$; (d) $N_{i}=50, \vartheta_{P M}=0.5$. The pheromone cloud is shown as gray gradient. Brood clusters placed in the arena centres are shown in dark gray.

To check for the influence of stigmergy, we explored changes in the mean length of each bulldozing episode for external ants. As expected, the mean bulldozing duration, measured from when an external ant bulldozed into the pheromone cloud to the terminal collision, decreased over time irrespective of values of $\vartheta_{P M}$ (Figure 6) due to the progressively higher frequency of encountering already placed stones. This behaviour is consistent with Franks and Deneubourg (1997), who implied that stone carrying time decays exponentially as the nest building progresses.

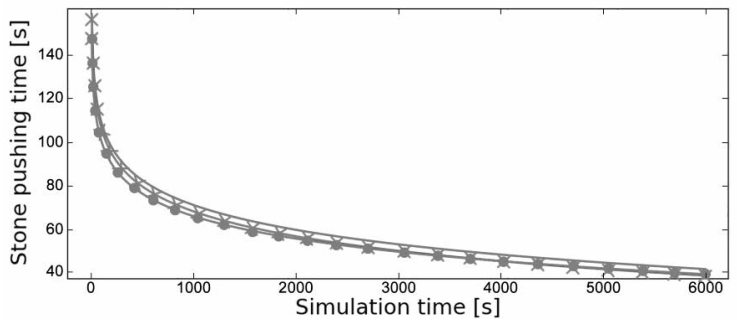

Figure 6: Fitted exponential models of mean bulldozing time measured for external ants between the moment they entered the pheromone cloud and the moment they dropped stones. $N_{i}=30, N_{e}=10$ and $\vartheta_{P M}=1.0$ (solid, $R^{2}=39.97 \%$ ), $\vartheta_{P M}=0.75$ (crosses, $\left.R^{2}=37.83 \%\right), \vartheta_{P M}=0.5$ (dots, $R^{2}=$ $28.04 \%$ ). (Note that the inherent stochasticity of the simulation means that the exponential model does not account for all of the variance in bulldozing times.)

\section{Physical Building Template Only}

We first explored building behavior in the absence of a pheromone building template.

The size of nests built by real ant colonies depended on the number of colony members (Franks and Deneubourg, 1997). Similarly, increasing the number of simulated internal ants, $N_{i}$, tended to increase the effective diameter of final structures (Figure 7a). Increasing colony size also increased the irregularity of the built structures, measured as the standard deviation of the number of stones found in eight regular conical sectors, each originating from the centre of the pheromone cloud (Figure 7b).

Both nest diameter and nest regularity were also dependent on the value of $\vartheta_{P M}$, i.e. on how far from the brood cluster internal ants were 'willing' to roam before turning back towards it. Only when $\vartheta_{P M}$ was higher than a specific threshold value, was a colony able to effectively encircle itself with stones.

Where $\vartheta_{P M}$ was high, internal ants were tightly clustered and regular circular nests were built relatively close to the central brood cluster. For runs with lower $\vartheta_{P M}$, internal ants spread out from the brood cluster, pushing the built structure out, but their lower density at the characteristic radius of the nest wall allowed gaps to form, compromising its regularity. For even lower values of $\vartheta_{P M}$, the internal ants were spread out to such an extent that they cease to be an effective physical template for building, allowing external ants to build much closer to the brood cluster. The critical value of $\vartheta_{P M}$ (below which the physical building template fails) varied inversely with $N_{i}$ as larger colonies could achieve sufficient density at greater distance from the brood cluster.

As $\vartheta_{P M}$ approached zero and the density of internal ants was minimised, many stones were left very near the middle of the pheromone cloud, since external ants were often able to bring them to the brood and there was a lower probability 


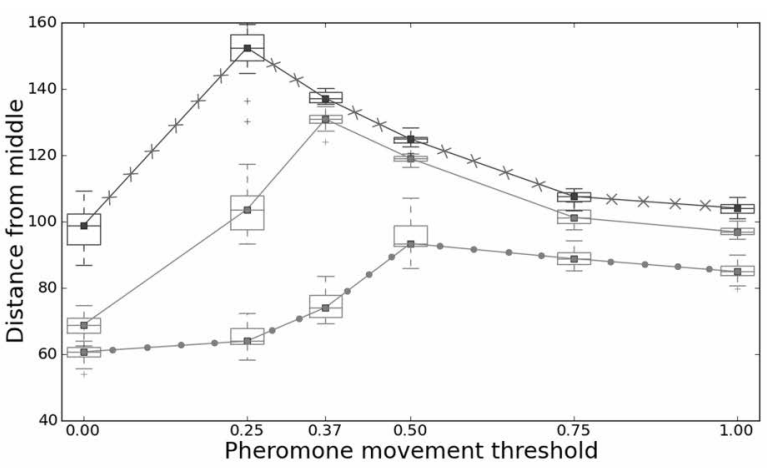

(a)

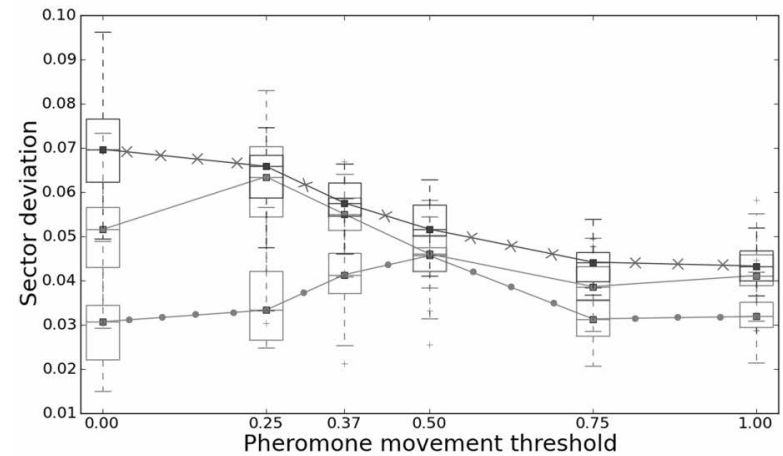

(b)

Figure 7: Influence of $\vartheta_{P M}$ on (a) average distance of stones from the middle of the pheromone cloud, and (b) standard deviation of the number of stones in eight regular conical sectors of the cloud for $N_{e}=10$ (all) and $N_{i}=10$ (dots), $N_{i}=30$ (solid), $N_{i}=50$ (crosses).

of an internal ant encountering and removing them. In these cases, built structures were packed close to the brood and were often very irregular.

The number of external ants also had a non-trivial influence on building behaviour. With the number of internal ants fixed at $N_{i}=30$ we explored the influence of increasing external ant numbers from 10 to 180 for two pheromone thresholds, $\vartheta_{P M}=0.5$ and $\vartheta_{P M}=1.0$. Increasing the number of external ants, $N_{e}$, from 10 to 30 caused nests to become smaller, as expected, since increased pressure from external ants tended to establish walls closer to the brood cluster (Figure 8a). However, contra to Hypothesis 1, further increases to $N_{e}$ gave rise to larger nests as a consequence of interference between external ants, some of which found themselves within the pheromone cloud during or after bulldozing, effectively augmenting the number of internal ants and amplifying the effect of their physical building template. Increasing the number of external ants, $N_{e}$, also tended to increase the amplification of their initial building sites, decreasing the regularity of built structures (Figure 8b).

In summary, this section has confirmed that model ants are able to achieve built structures the size and integrity of

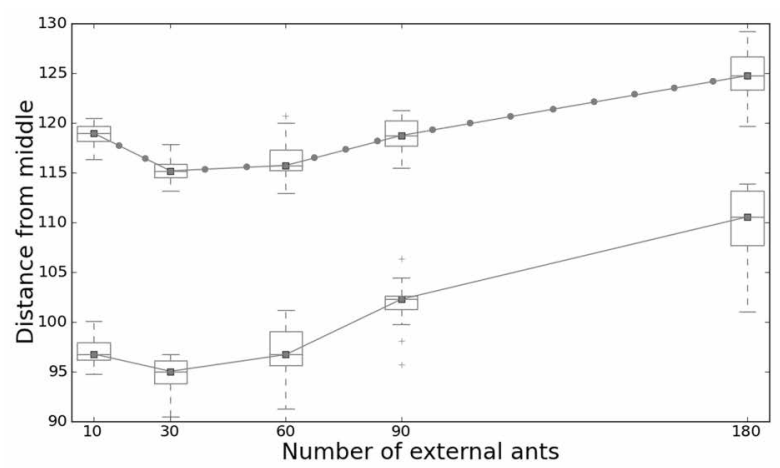

(a)

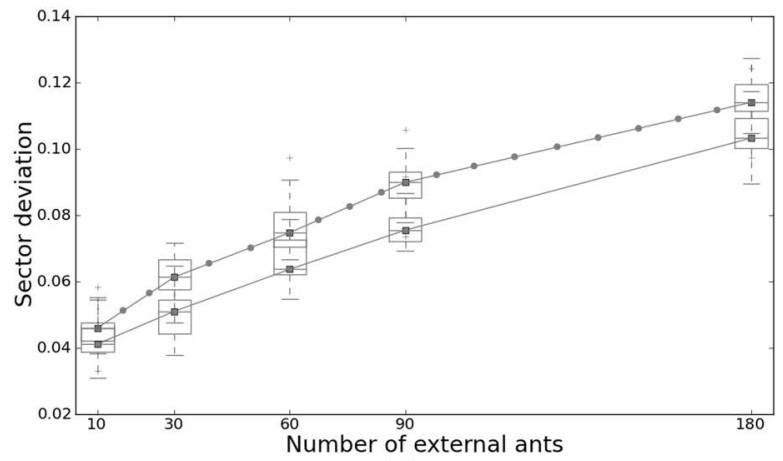

(b)

Figure 8: Influence of external ant number on (a) average distance of stones from the middle of the pheromone cloud, and (b) standard deviation of the number of stones in eight regular conical sectors of the cloud for $N_{i}=30$ (all) and $\vartheta_{P M}=0.5$ (dots), $\vartheta_{P M}=1.0$ (solid).

which reflect a complicated interaction between the size of a colony and the extent to which internal ants tend to wander from the brood. They are able to achieve these structures in the absence of a pheromone building template, i.e. pheromone-mediated dropping behaviour is not necessary for successful nest formation. In the next section we explore the influence of such a pheromone building template and assess entrance formation.

\section{Physical + Pheromone Building Templates}

The effect of employing a pheromone building template to encourage the dropping behaviour of internal ants (Equation 4) is depicted in Figure 9. Ants mostly tended to build smaller and more regular structures as dropping became more precisely tied to an internal ant's location within the pheromone cloud. This effect was more significant for larger colonies and those where internal ants roamed further from the brood cluster.

When the pheromone building template was used with large numbers of internal ants, e.g. $N_{i}=50$, the influence of pheromone on building initially caused stones to be dropped closer to the brood cluster, but nests were subsequently ex- 


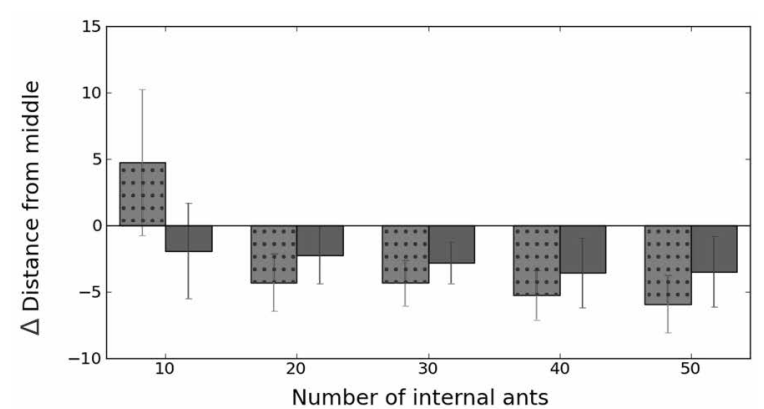

(a)

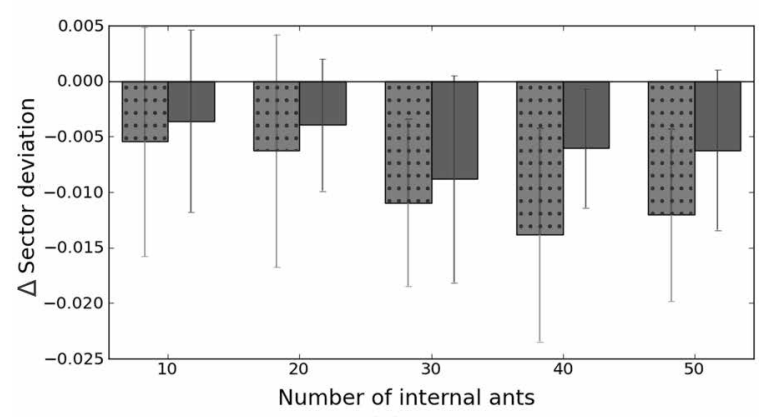

(b)

Figure 9: Difference between runs with and without pheromone building template in terms of (a) average distance of stones from the middle of the cloud where a negative number indicates smaller nests with pheromone building templates, and (b) standard deviation of the number of stones in eight conical sectors of the cloud where a negative number indicates more regular structures with the pheromone building templates, using $N_{e}=10$ with $\vartheta_{P M}=0.5$ (dots) and $\vartheta_{P M}=1.0$ (solid).

panded to some extent due to the pressure of internal ants within the walls.

By contrast, when the number of internal ants was small, e.g. $N_{i}=10$, and they remained close to the brood cluster $\left(\vartheta_{P M}=1.0\right)$, the pheromone building template had little effect since internal ants rarely roamed a significant distance from the brood cluster.

However, when the same small number of internal ants were allowed to roam further from the brood cluster $\left(\vartheta_{P M}\right.$ $=0.5$ ), whereas without a pheromone building template they failed to achieve a nest wall, tending to assemble many stones in the middle of the cloud, with a pheromone building template more regular structures were achieved at an increased average distance from the brood cluster. In this case, the pheromone building template made nest creation possible when it otherwise would not be, by encouraging a wall to be built closer to the brood.

\section{Entrance Formation}

Experimental runs were evaluated manually in order to categorise the final structures by number of entrances and

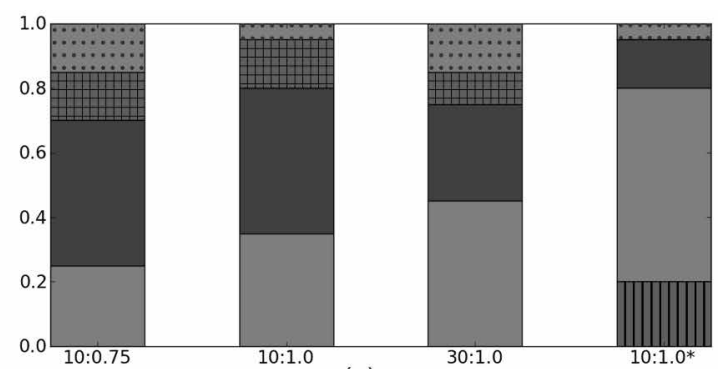

(a)

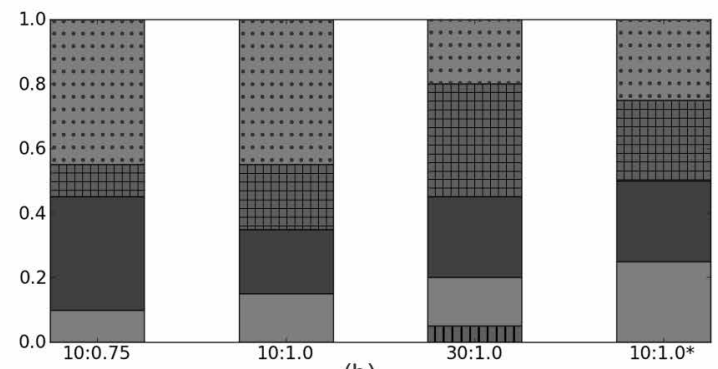

(b)

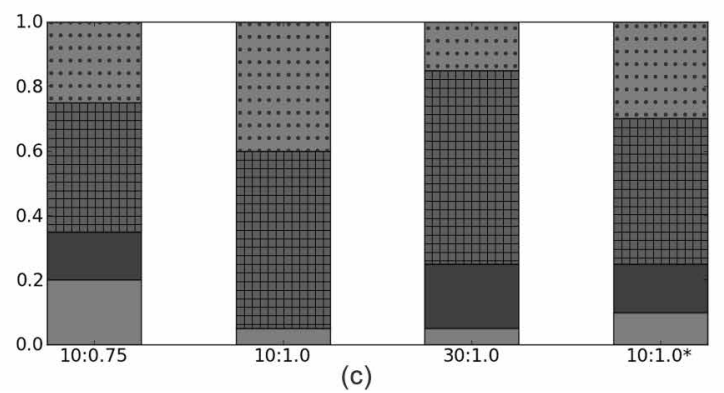

Figure 10: Proportion of nests with 0 (bars), 1 or 2 (light solid), 3 (dark solid) or more entrances (hatch) and irregular structures (dots) using (a) $N_{i}=10$, (b) $N_{i}=30$ and (c) $N_{i}=50$. The individual groups are labeled using pattern $N_{e}: \vartheta_{P M}$. A star (*) indicates that the pheromone building template was employed.

whether they could be considered nests at all (Figure 10). The most regular nests were built when 10 internal ants were used. Irregular nests (i.e. arrangements of stones that did not form a coherent structure at all) occurred only $5 \%$ of the time when $\vartheta_{P M}=1.0$ and $15 \%$ of the time when $\vartheta_{P M}=0.75$. The frequency of nests with only 1 or 2 entrances increased as the number of external ants increased and similarly when the pheromone building template was used. However, in the latter case, the ants also built a complete wall with no entrances at all in 4/20 runs.

A similar pattern of entrance formation was observed in colonies with 30 internal ants, although generally the frequency of irregular structures increased in comparison with the previous case. Furthermore, these larger colonies tended to build nests with three or more entrances more often, especially when $N_{e}=30$ (12/20 runs). A nest with no entrances 
was only built on one occasion when $N_{e}=30$.

The trend to create more entrances was even stronger for colonies with 50 internal ants. This was especially true when $\vartheta_{P M}=1.0$, in which case nests with four or more entrances formed in 11/20 runs. Interestingly, regularity of nests increased in comparison with colonies where $N_{i}=30\left(N_{i}=\right.$ 30 and $\vartheta_{P M}=0.75$ or $\vartheta_{P M}=1.0$, regular nests occurred in $11 / 20$ runs, while $N_{i}=50, \vartheta_{P M}=0.75$, in $15 / 20$ runs and $N_{i}=50, \vartheta_{P M}=1.0$, in 12/20 runs). Nest regularity increased slightly in the experiments with pheromone building templates $\left(N_{e}=10, \vartheta_{P M}=1.0\right.$ regular nests in $12 / 20$ runs and in 14/20 runs during the pheromone building template experiments), although regular nests were the most frequent when $N_{e}=30$ and $\vartheta_{P M}=1.0$ (16/20 runs).

\section{Controlling Nest Shape}

Standard structures created by ants with a single pheromone cloud were circular. In the following set of experiments, the pheromone cloud diameter was decreased from 300 units to 150 units and a number of clouds with brood clusters at their centres were arranged in order to create nests of different shapes.

Three experimental setups were created: a) rectangle: two clouds were horizontally aligned and the distance of their centres was set to 75 units, b) triangle: three clouds, the centers of which formed the vertices of a triangle with sides 75 units long, and c) square: four clouds, the centers of which formed corners of a square with 75 units side length. The number of external ants was 10, while there were always 10 internal ants per pheromone cloud. The value of $\vartheta_{P M}$ was set to 1.0 , since the previous experiments showed that the most regular nests were built with this value (Figures 7-10). Note that ants were unable to distinguish amongst the different sources of pheromone.

The final positions from 20 runs in each experiment were amalgamated in order to generate contour plots (Figure 11). In each experiment, the desired shape was always achieved, although there were no sharp corners as walls naturally curved around the boundaries of the individual pheromone clouds. Once again, using the pheromone building template facilitated creation of more regular structures, although it was not required to achieve the desired shapes.

One or two entrances usually formed along the shorter edges of rectangular structures. The triangular nests had one to three small entrances that could be found near the vertices. Entrances in square nests were usually more numerous and formed both along the edges and in the corners. Probably due to their size, square structures had the least regular distribution of stones in their walls.

\section{Discussion}

Nest formation by simulated ants was tested in a number of different scenarios. The final circular structures, as well as the process by which they were built were comparable to real

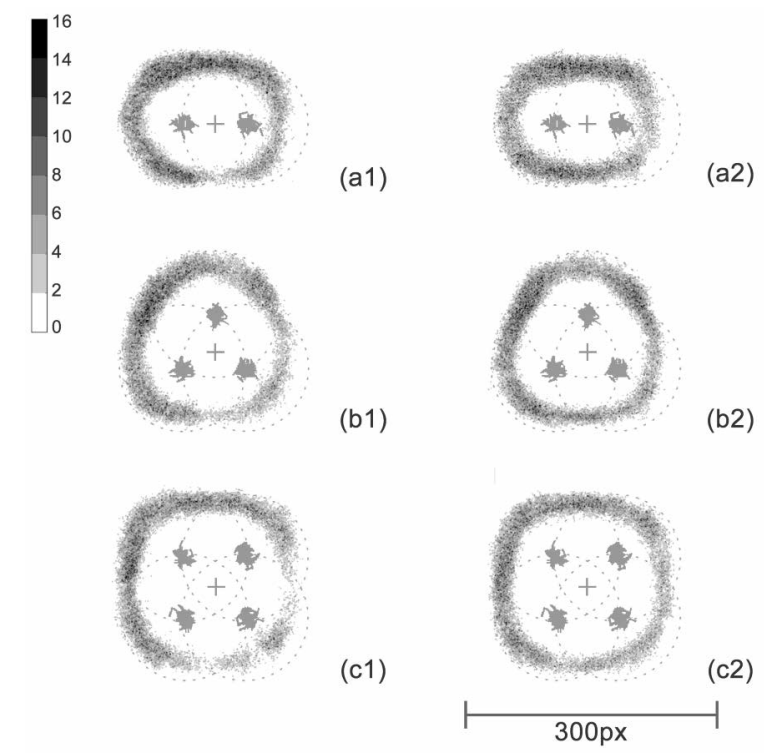

Figure 11: Contour plots of nests created during the (a) rectangle, (b) triangle, and (c) square experiments. Results from experiments (1) without a pheromone building template, and (2) with such a template are shown. Pheromone clouds are represented by dotted circles. Crosses show arena centres, with brood clusters around them.

and previously simulated ants (Franks et al., 1992; Theraulaz et al., 2003) across a wide parameter space. Usually, the ants initially created a number of stone heaps that were gradually extended and connected together, while nest entrances remained clear throughout the process. Adding more external ants initially caused gaps in the nest wall to be created and destroyed, with stable entrances appearing only later in the simulations.

This building behaviour was more similar to that of real ants that clear a cavity of stones and create a number of progressively joined heaps rather than of those that bring stones from outside of the building site and gradually form a Cshaped nest with only one entrance (Franks et al., 1992). It is possible that in the latter case, external ants carry stones towards the nest from one direction, rather than from all directions as was the case in the simulated arena, or that they find stones further away from the nest, causing a slower stone intake rate and thus different wall formation dynamics.

The nest size varied as the number of internal ants increased, confirming the assumption of Hypothesis 1, although regularity of the structures decreased when they became large. The differences in nest size occurred due to variations in ant movement as large colonies required more space to spread out. Similarly, larger structures were formed when movement of ants within the pheromone cloud became less restricted by varying the $\vartheta_{P M}$ parameter.

On the other hand, the assumption of Hypothesis 1 that 
larger numbers of external ants $N_{e}$ would cause higher pressure and lead to the creation of smaller nests was only true when $N_{e}$ was relatively low. The nests actually became larger and less regular when $N_{e} \geq 90$. This surprising increase in nest diameter occurred because more external ants could be found inside the pheromone cloud, adding to the size of the physical template formed by the internal ants and brood. Nest regularity decreased as higher numbers of external ants assembled stones more rapidly, increasing the probability of amplifying any initial building sites.

Use of the pheromone cloud as a template for building improved nest regularity (Figures 9, 10, 11). Furthermore, the resulting structures were smaller as the gradient of pheromone concentration interfered with the effect of ant movement, confirming Hypothesis 2. This effect could not be observed with only 10 internal ants when they were restricted to remain very close to the centre of the pheromone cloud as they could not reach places influenced by the pheromone building template.

The fact that the pheromone template was not required for successful nest construction agrees with the assumption of Franks and Deneubourg (1997) who understood pheromone as simply a cue for ants to orient themselves within the nest. Allowing pheromone to influence stone deposition directly (e.g. Theraulaz et al., 2003) thus seems unnecessary.

The assumption of Hypothesis 3, that nest entrances would form when internal ant movement is more constrained $\left(\vartheta_{P M}\right.$ is high), was partially supported (Figure 10$)$. When the number of internal ants was low, $N_{i}=10$, more regular structures were produced for $\vartheta_{P M}=1.0$ compared to $\vartheta_{P M}$ $=0.75$. However, the effect of $\vartheta_{P M}$ was not apparent when $N_{i}=30$ and was reversed when $N_{i}=50$, i.e., the influence of $\vartheta_{P M}$ on nest regularity varied with colony size. On the other hand, use of the pheromone building template always improved nest regularity, as predicted.

Finally, it was shown that non-circular nest shapes can be created when multiple pheromone clouds are arranged together (Figure 11), as predicted by Hypothesis 4 . The clouds needed to be small enough so that there were enough stones available to create the final shapes and also suitably close to each other so that internal ants could travel between them.

\section{Conclusion}

In conclusion, this work has helped to understand the building behaviour of the ant Leptothorax tuberointerruptus and to answer questions about the roles of colony size and pheromone-mediated behaviour in the building process. It is clear from the results presented here that even in the very idealised and simple scenario that we explore, the interactions that give rise to built structures are subtle and complex. We also show that this simple behavioural algorithm could perhaps be applied with cheap robotic ants to create structures beyond the circles achieved by L. tuberointerruptus. Extensions to the model include adding agent-generated pheromone gradients, and applying the revealed principles of nest morphogenesis to the decentralised construction of more complicated heterogeneous architectures.

Acknowledgements: This work was supported by an EPSRC Doctoral Training Centre grant (EP/G03690X/1).

\section{References}

Bonabeau, E., Theraulaz, G., Deneubourg, J.-L., Franks, N. R., Rafelsberger, O., Joly, J.-L., and Blanco, S. (1998). A model of the emergence of pillars, walls and royal chambers in termite nests. Philosophical Transactions of the Royal Society of London, Series B, 353:1561-1576.

Bourg, D. M. and Seemann, G. (2004). AI for Game Developers. O'Reilly Media.

Bullock, S., Ladley, D., and Kerby, M. (2012). Wasps, termites, and waspmites: Distinguishing competence from performance in collective construction. Artificial Life, 18(3):267-90.

Di Marzo Serugendo, G., Gleizes, M.-P., and Karageorgos, A. (2011). Self-organising systems. In Di Marzo Serugendo, G., Gleizes, M.-P., and Karageorgos, A., editors, Self-organising Software, Natural Computing Series, pages 7-32. Springer.

Franks, N. and Deneubourg, J. (1997). Self-organizing nest construction in ants: Individual worker behaviour and the nest's dynamics. Animal Behaviour, 54(4):779-96.

Franks, N., Wilby, A., Silverman, B., and Tofts, C. (1992). Selforganizing nest construction in ants: Sophisticated building by blind bulldozing. Animal Behaviour, 44:357-375.

Holland, O. and Melhuish, C. (1999). Sorting in collective robotics. In Langton, C. G. and Shimohara, T., editors, Artificial Life $V$, pages 173-202. MIT Press.

Jeanne, R. L. and Bouwma, A. M. (2002). Scaling in nests of a social wasp: A property of the social group. The Biological Bulletin, 202(3):289-95.

Ladley, D. and Bullock, S. (2004). Logistic constraints on 3D termite construction. In Dorigo, M., Birattari, M., Blum, C., Gambardella, L. M., Mondada, F., and Stutzle, T., editors, The Fourth International Workshop on Ant Colony Optimisation, pages 178-189. Springer, Berlin.

Ladley, D. and Bullock, S. (2005). The role of logistic constraints on termite construction of chambers and tunnels. Journal of Theoretical Biology, 234:551-564.

Mason, Z. (2002). Programming with stigmergy: Using swarms for construction. In Standish, R., Bedau, M. A., and Abbass, H. A., editors, Artificial Life VIII, pages 371-374. MIT Press.

Parker, C. A. C. and Zhang, H. (2006). Collective robotic site preparation. Adaptive Behaviour, 14(1):5-19.

Theraulaz, G., Bonabeau, E., and Deneubourg, J.-L. (1998). The origin of nest complexity in social insects. Complexity, $3(6): 15-25$.

Theraulaz, G., Gautrais, J., Camazine, S., and Deneubourg, J.-L. (2003). The formation of spatial patterns in social insects: from simple behaviours to complex structures. Philosophical Transactions of the Royal Society of London, Series A, 361(1807):1263-82. 